\title{
Seminare / Séminaires / Seminari
}

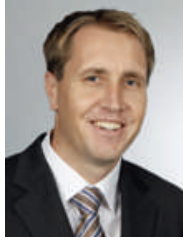

René Häller,

Geschäftsführer FMH Consulting Services

\section{Praxiseröffnung/-übernahme}

Das Seminar richtet sich an Ärztinnen und Ärzte, die vor einer Praxiseröffnung (Einzel-/ Gruppenpraxis), dem Einstieg in eine Gruppenpraxis oder vor einer Praxisübernahme stehen.

\section{Themen}

- Juristische Aspekte (Praxisbewilligung, Zulassung zur Sozialversicherung, Vertragswesen)

- Gesellschaftsformen/Ehe- und Erbrecht (Privat-/Geschäftsvermögen, Güterstand, Erbschaftsplanung)

- Praxiseinrichtung (Inneneinrichtung, Kostenberechnung)

- Praxisadministration (Leistungserfassungsund Abrechnungssysteme)

- Bewertung einer Arztpraxis (Berechnung Inventarwert und Goodwill als Verhandlungsbasis)

- Finanzierung der Arztpraxis (Businessplan, Kredite, Absicherungsmöglichkeiten)

- Versicherungen/Vorsorge/Vermögen (Personen- und Sachversicherungen, Vorsorgeplanung)

\section{Sponsoren}

Die Kosten werden durch diverse Sponsoren (siehe www.fmhservices.ch) gedeckt.

\begin{tabular}{lll}
\hline Daten & & \\
\hline K01 & $\begin{array}{l}\text { Donnerstag, } \\
\text { 3. März 2016 } \\
\text { 09.00-16.30 Uhr }\end{array}$ & $\begin{array}{l}\text { Zürich } \\
\text { Kolkshaus }\end{array}$ \\
& $\begin{array}{l}\text { Donnerstag, } \\
\text { 12. Mai 2016 } \\
\text { 16.00-20.30 Uhr }\end{array}$ & $\begin{array}{l}\text { St. Gallen } \\
\text { K03 }\end{array}$ \\
& $\begin{array}{l}\text { Donnerstag, } \\
\text { 9. Juni 2016 }\end{array}$ & Bern \\
K04 & $\begin{array}{l}\text { 09.00-16.30 Uhr } \\
\text { Donnerstag, }\end{array}$ & Schmiedstube \\
& $\begin{array}{l}\text { 1. September 2016 } \\
\text { 09.00-16.30 Uhr } \\
\text { K05 }\end{array}$ & Zürich \\
& $\begin{array}{l}\text { Donnerstag, } \\
\text { 3. November 2016 } \\
\text { 09.00-16.30 Uhr }\end{array}$ & Basel \\
& &
\end{tabular}

\section{Praxisübergabe/-aufgabe}

Das Seminar richtet sich an Ärztinnen und Ärzte, die ihre Praxis an einen Partner oder Nachfolger übergeben oder liquidieren wollen. Idealtermin:

Obwohl rund $40 \%$ aller Ärztinnen und Ärzte früher oder später eine eigene Arzt-/Gruppenpraxis führen und nebst dem medizinischen Know-how auch jenes eines Unternehmers benötigen, wird im Verlauf des Medizinstudiums die Thematik der Unternehmensführung kaum behandelt. Die FMH Services schliessen diese Lücke und kümmern sich um die betriebswirtschaftlichen Anliegen ihrer Genossenschafter unter anderem mit speziell konzipierten Seminaren, an denen jährlich rund 750 Ärztinnen und Ärzte teilnehmen. Die grösstenteils kostenlosen Seminare vermitteln wichtiges Grundwissen für den Einstieg in die eigene Arzt-/Gruppenpraxis, deren Führung und Betrieb. Die Teilnehmer werden auf den Umgang und die Zusammenarbeit mit Behörden und Fachspezialisten wie Treuhänder, Versicherungs- und Finanzberater, Kreditberater, Juristen, Architekten usw. vorbereitet. Zu den einzelnen Themen rund um die Praxiseröffnung und -führung referieren ausgewiesene Experten der FMH Services.

Bien qu'environ $40 \%$ de tous les médecins ouvrent tôt ou tard leur propre cabinet médical individue ou de groupe et qu'en plus de leur savoir-faire médical, ils aient besoin de connaissances en gestion d'entreprise, cette thématique est peu abordée lors des études. FMH Services, qui s'occupent de la gestion d'entreprise de ses membres, comblent cette lacune en proposant, entre autres, des séminaires spécifiques qui réunissent chaque année quelque 750 médecins. Dans ces séminaires, gratuits pour la plupart, les participants peuvent acquérir les bases nécessaires pour ouvrir leur propre cabinet médical individuel ou de groupe. Ils obtiennent également des conseils sur la manière de collaborer avec les autorités ou avec des spécialistes tels que des experts-comptables, des conseillers financiers, en assurance ou en crédits bancaires, ainsi que des juristes, des architectes et autres. Ces différents thèmes sont abordés par des experts qualifiés de FMH Services.

5-10 Jahre vor geplanter Übergabe oder allfälliger Liquidation (aus steuertechnischen und vorsorgeplanerischen Gründen).

\section{Themen}

- Praxispartner- oder Nachfolgesuche (projektorientiertes Vorgehen in der Nachfolgeplanung)

- Juristische Aspekte (Praxisübergabevertrag, allg. Vertragswesen, Übergabe der Krankengeschichten)

- Bewertung einer Arztpraxis (Berechnung Inventarwert und Goodwill als Verhandlungsbasis)

- Versicherungen/Vorsorge/Vermögen (Übergabe/Auflösung von Versicherungsverträgen, Pensions- und Finanzplanung)

- Steuern (Steueraspekte bei der Praxisübergabe oder Liquidation: Optimierung der steuerlichen Auswirkungen, Liquidations- und Grundstückgewinnsteuer, Bestimmung des optimalen Übergabe-/Aufgabezeitpunktes)

\section{Sponsoren}

Die Kosten werden durch diverse Sponsoren (siehe www.fmhservices.ch) gedeckt.

\begin{tabular}{|c|c|c|}
\hline \multicolumn{3}{|c|}{ Daten } \\
\hline K06 & $\begin{array}{l}\text { Donnerstag, } \\
\text { 10. März } 2016 \\
\text { 13.30-18.00 Uhr }\end{array}$ & $\begin{array}{l}\text { Zürich } \\
\text { Volkshaus }\end{array}$ \\
\hline K07 & $\begin{array}{l}\text { Donnerstag, } \\
\text { 19. Mai } 2016 \\
\text { 16.00-20.30 Uhr }\end{array}$ & $\begin{array}{l}\text { St. Gallen } \\
\text { Hotel Einstein }\end{array}$ \\
\hline K08 & $\begin{array}{l}\text { Donnerstag, } \\
\text { 16. Juni } 2016 \\
\text { 13.30-18.00 Uhr }\end{array}$ & $\begin{array}{l}\text { Bern } \\
\text { Schmiedstube }\end{array}$ \\
\hline K09 & $\begin{array}{l}\text { Donnerstag, } \\
\text { 8. September } 2016 \\
13.30-18.00 \text { Uhr }\end{array}$ & $\begin{array}{l}\text { Zürich } \\
\text { Volkshaus }\end{array}$ \\
\hline K10 & $\begin{array}{l}\text { Donnerstag, } \\
\text { 10. November } 2016 \\
13.30-18.00 \text { Uhr }\end{array}$ & $\begin{array}{l}\text { Basel } \\
\text { Hotel Victoria }\end{array}$ \\
\hline
\end{tabular}

Finanz- und Steuerplanung

Das Seminar richtet sich an Ärztinnen und Ärzte, die vor einer Praxiseröffnung oder Praxisübernahme stehen oder bereits praxistätig sind.

\section{Themen}

- Finanzplanung (Businessplan, buchhalterische Massnahmen vor Praxiseröffnung/ -übernahme, Standard-Kontenplan, doppelte Buchhaltung, EDV-unterstützte Buchführungslösung)

- Steuern (Steueraspekte bei Eintritt in die Selbständigkeit, Steuerfallen und Steuerrisiken, optimierte Steuerplanung)

\section{Kosten}

Für FMH Services-Mitglieder kostenlos.

\begin{tabular}{lll}
\hline Daten & & \\
\hline K11 & $\begin{array}{l}\text { Donnerstag, } \\
\text { 17. März 2016 }\end{array}$ & Zürich \\
& $\begin{array}{l}\text { 13.30-18.00 Uhr } \\
\text { K12 }\end{array}$ & \\
& $\begin{array}{l}\text { Donnerstag, } \\
\text { 15. September 2016 } \\
\text { 13.30-18.00 Uhr }\end{array}$ & Bern \\
& &
\end{tabular}

\section{Praxiscomputer-Workshop}

Der Workshop richtet sich an Ärztinnen und Ärzte, die vor einer Praxiseröffnung oder Praxisübernahme stehen oder bereits praxistätig sind.

\section{Themen}

- Anforderungen an ein Praxisinformationssystem (Einführung)

- Evaluationsprozess (projektorientiertes Vorgehen in der Evaluation eines Praxisinformationssystems)

- Präsentation von sechs führenden Praxisinformationssystemen (Leistungserfassung, 
elektronisches Abrechnen unter Einbezug der TrustCenter, Agendaführung, Statistiken, Laborgeräteeinbindung, elektronische Krankengeschichte, Finanzbuchhaltungslösungen usw.)

Kosten

Für FMH Services-Mitglieder kostenlos.

\begin{tabular}{lll}
\hline Daten & & \\
\hline K13 & $\begin{array}{l}\text { Donnerstag, } \\
\text { 24. März 2016 } \\
\text { 13.30-18.00 Uhr }\end{array}$ & $\begin{array}{l}\text { Zürich } \\
\text { Technopark }\end{array}$ \\
K14 & $\begin{array}{l}\text { Donnerstag, } \\
\text { 30. Juni 2016 } \\
\text { 13.30-18.00 Uhr }\end{array}$ & Bern \\
K15 & $\begin{array}{l}\text { Dennerstag, } \\
\text { 24. November 2016 }\end{array}$ & Olten \\
& Stadtheater \\
& 13.30-18.00 Uhr & \\
\end{tabular}

Going paperless - Intensivkurs

Der Workshop richtet sich an Ärztinnen und Ärzte, die ihre Praxis mit digitalen Krankengeschichten führen wollen.

\section{Themen}

- Struktur und Organisation (Strategische Ausrichtung, Vor- und Nachteile der Digitalisierung, Geschäftsprozesse und Informationsfluss)

- Erstellen eines Anforderungskataloges (Lastenheft) und seine Verwendungszwecke

- Das Führen der elektronischen Krankengeschichte (Aufbau und Struktur)

\section{Kosten}

200 CHF (inkl. Kursunterlagen und Verpflegung).

\begin{tabular}{lll}
\hline Daten & & \\
\hline K92 & Donnerstag, & Zürich \\
& 21. April 2016 & Kongresshaus \\
09.00-16.30 Uhr & \\
K93 & $\begin{array}{l}\text { Donnerstag, } \\
\text { 22. September 2016 } \\
\text { 09.00-16.30 Uhr }\end{array}$ & Sern \\
&
\end{tabular}

\section{Gruppenpraxis}

Das Seminar richtet sich an in Ausbildung stehende Ärztinnen und Ärzte, die sich einer Gruppenpraxis anschliessen wollen, sowie an praxistätige Ärztinnen und Ärzte, die ihre Einzelpraxis an eine Gruppenpraxis anschliessen wollen.

\section{Themen}

- Strategie (Ziele der Gruppenpraxis; Gestaltung des Angebots)

- Unternehmer (Zusammensetzung des Teams; Verhaltensregeln, finanzielle Beteiligung und Entschädigungsmodelle)

- Finanzen und Recht (Versicherung, Vorsorge und Vermögen; Rechtsform, Finanzen, Steuern)

- Standort, Praxisobjekt (Anforderungen an Standort; Konkurrenzanalyse; Praxiseinrichtung, Kosten)
- Personal (Qualifikationen; Gesetze, Reglemente, Verträge)

- Führung und Organisation (Struktur und Abläufe; Aufgaben, Verantwortungen, Kompetenzen)

- EDV und Administration (Anforderungen an Praxisinformatik; Evaluation)

- Praxisbericht (Erfahrungsbericht eines Arztes, Mitgründer einer Gruppenpraxis aus der Region)

\section{Sponsoren}

Die Kosten werden durch diverse Sponsoren (siehe www.fmhservices.ch) gedeckt.

\begin{tabular}{lll}
\hline Daten & & \\
\hline K80 & $\begin{array}{l}\text { Donnerstag, } \\
\text { 16. Juni 2016 } \\
\text { 13.30-18.00 Uhr }\end{array}$ & St. Gallen \\
K81 & $\begin{array}{l}\text { Donnerstag, } \\
\text { 17. November 2016 } \\
\text { 13.30-18.00 Uhr }\end{array}$ & Bern \\
& Schmiedstube \\
&
\end{tabular}

\section{Röntgen in der Arztpraxis}

Das Seminar richtet sich an Ärztinnen und Ärzte, die vor einer Praxiseröffnung oder Praxisübernahme stehen oder bereits praxistätig sind.

\section{Themen}

- Rentabilität Röntgen in der Arztpraxis

- Evaluation und Beschaffung neuer oder gebrauchter Anlagen

- Möglichkeiten der Umrüstung von analogen zu digitalen Anlagen

- Vor- und Nachteile analoger und digitaler Systeme

- Komplette Marktübersicht mit Preisen und Leistungskomponenten

Kosten

Für FMH Services-Mitglieder kostenlos.

\begin{tabular}{lll}
\hline Daten & & \\
\hline K16 & Donnerstag, & Niederscherli \\
& 25. August 2016 & WIROMA AG \\
& $09.30-16.00 \mathrm{Uhr}$ &
\end{tabular}

Tarifwerk TARMED - Einführungskurs

Das Seminar richtet sich an Ärztinnen und Ärzte mit bestehender Praxis und an solche, die kurz vor einer Praxiseröffnung oder Praxisübernahme stehen. Der Einführungskurs vermittelt den Kursteilnehmern die Grundlagen des Tarifwerkes TARMED.

\section{Themen}

- Fakten (gesetzliche und vertragliche Grundlagen)

- Struktur (Tarifbrowser, Grundstruktur, Regelhierarchie, Leistungsblöcke, Leistungsgruppen)

- Generelle Interpretationen ("Allgemeine Grundleistungen", "Hauptleistungen, Zuschlagsleistungen», "Nichtärztliche Leistungserbringung» usw.)
- Parameter einer Tarifposition ("Quantitative und Qualitative Dignität», "Ärztliche Leistung AL», "Assistenz», «Raumbelegung» usw.)

- Tarifpositionen aus dem Kapitel 00 Grundleistungen

- Praxislabor und Präsenzdiagnostik

- Organisationen und Informationsquellen

\section{Kosten}

150 CHF (inkl. Kursunterlagen). Die Referentenkosten sind durch Sponsoren gedeckt.

\begin{tabular}{lll}
\hline Daten & & \\
\hline K60 & $\begin{array}{l}\text { Dienstag, } \\
\text { 8. März 2016 } \\
\text { 13.30-16.45 Uhr }\end{array}$ & $\begin{array}{l}\text { Olten } \\
\text { Hotel Arte }\end{array}$ \\
K61 & $\begin{array}{l}\text { Dienstag, } \\
\text { 10. Mai 2016 }\end{array}$ & $\begin{array}{l}\text { Olten } \\
\text { Hotel Arte }\end{array}$ \\
& $\begin{array}{l}\text { 13.30-16.45 Uhr } \\
\text { K62 }\end{array}$ & \\
& $\begin{array}{l}\text { Dienstag, } \\
\text { 13. September 2016 } \\
\text { 13.30-16.45 Uhr }\end{array}$ & Olten \\
& &
\end{tabular}

\section{Praxismarketing für Ärzte}

Das Seminar richtet sich an Ärztinnen und Ärzte, welche vor einer Praxiseröffnung stehen oder bereits eine Praxis führen und diese aktiv bewerben wollen.

Themen

- Gestaltung der Praxis aus Marketingsicht Umgang mit Patienten am Telefon und Empfang

- Praxiswebseite

- Kommunikation - Kompetenz, Vertrauen visualisieren und kommunizieren

Kosten

Für FMH Services-Mitglieder kostenlos.

\begin{tabular}{lll}
\hline Daten & & \\
\hline K63 & $\begin{array}{l}\text { Donnerstag, } \\
\text { 10. März 2016 }\end{array}$ & $\begin{array}{l}\text { Bauma } \\
\text { prama GmbH }\end{array}$ \\
& 13.30-18.00 Uhr & \\
K64 & Mittwoch, & Oberkirch \\
& 16. November 2016 & Marble \\
& 13.30-18.00 Uhr & Concept GmbH
\end{tabular}

Telefonseminar für MPAs

(bzw. Praxisteam)

Das Seminar richtet sich an MPAs mit telefonischem Kundenkontakt sowie Auszubildende, die zum professionellen Telefonieren angeleitet werden sollen.

Themen

- Die medizinische Praxisassistentin als Visitenkarte der Praxis

- Image der Arztpraxis. MPAs repräsentieren die Unternehmenskultur, organisieren die Praxis und sind somit ein wesentlicher Bestandteil für den Unternehmenserfolg.

- Bedeutung des ersten Telefonkontakts

- Richtig telefonieren - eine Anleitung 


\section{Kosten}

300 CHF (inkl. Kursunterlagen und Verpflegungen)

Praxisteams erhalten einen Rabatt von $20 \%$ pro Teilnehmer/in.

\begin{tabular}{lll}
\hline Daten & & \\
\hline K65 & Mittwoch, & Zürich \\
& 16. März 2016 & Volkshaus \\
K66 & $\begin{array}{l}\text { M.00-16.30 Uhr } \\
\text { Mittwoch, }\end{array}$ & \\
& $\begin{array}{l}\text { 9. November 2016 } \\
\text { 09.00-16.30 Uhr }\end{array}$ & Schmiedstube \\
&
\end{tabular}

\section{Kommunikation mit Patienten}

für MPAs (bzw. Praxisteam)

Das Seminar richtet sich an MPAs sowie Auszubildende, die zu einer professionelleren Kommunikation mit Patienten (auch schwierigen) angeleitet werden sollen und sich in der Kommunikation im Team verbessern möchten.

\section{Themen}

- Kommunikative Techniken (aktives Zuhören, 4-Ohren-Prinzip)

- Kundenorientierte Kommunikation

- Eigenes Konfliktverhalten

- Positiver Umgang mit Reklamationen und schwierigen Gesprächssituationen

\section{Kosten}

300 CHF (inkl. Kursunterlagen und Verpflegungen)

Praxisteams erhalten einen Rabatt von $20 \%$ pro Teilnehmer/in.

\begin{tabular}{lll}
\hline Daten & & \\
\hline K67 & Mittwoch, & Zürich \\
& 9. März 2016 & Volkshaus \\
K68 & $\begin{array}{l}\text { Mittwoch, } \\
\text { K6.30 Uhr }\end{array}$ & \\
& 7. September 2016 & Bern \\
& 09.00-16.30 Uhr &
\end{tabular}

\section{Anmeldung und Auskunft}

www.fmhservices.ch oder FMH Consulting Services, Cornelia Fuchs, Tel. 0419250077

\section{Hinweis}

Bei sämtlichen Seminaren, bei denen die Kosten teilweise oder gänzlich von Seminarsponsoren gedeckt werden, werden die Teilnehmeradressen den jeweiligen Sponsoren zur Verfügung gestellt.

\section{Annullierungsbedingungen}

Bei Abmeldungen oder Fernbleiben werden folgende Unkostenbeiträge erhoben:

- 50 CHF pro Person ab 14 Tage vor Seminarbeginn;

- 100 CHF pro Person ab 7 Tage vor Seminarbeginn oder Fernbleiben.
Ouverture et reprise d'un cabinet médical

Le séminaire est destiné aux médecins sur le point d'ouvrir un cabinet médical (individuel ou de groupe), de joindre un cabinet de groupe ou de reprendre un cabinet existant.

\section{Contenu}

- Aspects juridiques (contrats en général, autorisations, admission à pratiquer à la charge de l'assurance sociale, dossier patients)

- Business plan (préparation du plan de financement et crédit d'exploitation, financement par la banque)

- Aménagement (implantation, projet et concept d'aménagement, choix du mobilier, budget)

- Estimation d'un cabinet (inventaire et goodwill)

- Laboratoire

- Administration d'un cabinet médical

- Assurances

- Passage du statut de salarié à celui d'indépendant

- Fiscalité

\section{Sponsors}

Les coûts sont pris en charge par divers sponsors (voir www.fmhservices.ch).

\begin{tabular}{|c|c|c|}
\hline \multicolumn{3}{|c|}{ Dates } \\
\hline \multirow[t]{3}{*}{ K20 } & Jeudi & Lausanne \\
\hline & 3 mars 2016 & World Trade \\
\hline & $13 \mathrm{~h} 30-18 \mathrm{~h} 30$ & Center \\
\hline \multirow[t]{3}{*}{ K21 } & Jeudi & Genève \\
\hline & 2 juin 2016 & Crowne Plaza \\
\hline & $13 \mathrm{~h} 30-18 \mathrm{~h} 30$ & \\
\hline \multirow[t]{3}{*}{ K22 } & Jeudi & Lausanne \\
\hline & 1 septembre 2016 & World Trade \\
\hline & $13 \mathrm{~h} 30-18 \mathrm{~h} 30$ & Center \\
\hline
\end{tabular}

K23

Jeudi
3 novembre 2016
13h30-18h30

Genève

$13 \mathrm{~h} 30-18 \mathrm{~h} 30$

Remise et cessation d'un cabinet médical

Le séminaire s'adresse aux médecins désirant remettre un cabinet médical à un associé ou à un successeur ou qui doivent fermer leur cabinet médical. Idéalement 5-10 ans avant la remise/cessation prévue (pour des questions de taxation et prévoyance)

\section{Contenu}

- Recherche active d'un successeur/associé

- Aspects juridiques (contrat de remise, contrats en général, dossiers médicaux)

- Estimation d'un cabinet (calcul de l'inventaire et du goodwill comme base de négociations)

- Assurances/prévoyance/patrimoine (remise/résiliation des contrats d'assurances, formes de prévoyance, planification de la retraite et des finances)

- Conséquences fiscales d'une remise ou d'une cessation (optimisation de l'impact fiscale lors d'une remise/cessation, impôt sur les bénéfices et gains immobiliers, détermination de la date optimale pour la remise/ cessation.

\section{Sponsors}

Les coûts sont pris en charge par divers sponsors (voir www.fmhservices.ch).

\begin{tabular}{lll}
\hline Dates & & \\
\hline K24 & Jeudi & Lausanne \\
& 19 mai 2016 & World Trade \\
& 13h30-18h00 & Center
\end{tabular}

$$
\begin{aligned}
& \text { Jeudi } \\
& 10 \text { novembre } 2016 \\
& 13 \text { h30-18h00 }
\end{aligned}
$$

Genève

Crowne Plaza

\section{Cabinet de groupe}

Le séminaire s'adresse aux médecins en formation voulant exercer leur future activité en cabinet de groupe et aux libres praticiens qui souhaitent affilier leur cabinet individuel à un cabinet de groupe.

\section{Contenu}

- Stratégie (objectifs du cabinet de groupe; structure de l'offre de prestations)

- Entrepreneurs (composition de l'équipe; règles de conduite; participation financière et modèles de rémunération)

- Finances \& droit (assurances, prévoyance et patrimoine; forme juridique, finances et impôts)

- Lieu d'implantation \& immobilier (exigences locales; analyse de la situation concurrentielle; aménagement du cabinet et coûts)

- Ressources humaines (qualifications; lois, règlements et contrats)

- Direction \& organisation (structure et processus; tâches, responsabilités et compétences)

- Informatique \& administration (attentes en matière de système informatique pour le cabinet; évaluation)

- Rapport d'expérience (rapport de l'expérience d'un médecin, co-fondateur d'un cabinet de groupe de la région)

\section{Sponsors}

Les coûts sont pris en charge par divers sponsors (voir www.fmhservices.ch). 


\begin{tabular}{lll}
\hline Date & & \\
\hline K85 & Jeudi & Genève \\
& $\begin{array}{l}\text { 21 avril 2016 } \\
\text { 13h30-18h00 }\end{array}$ & Crowne Plaza \\
K86 & $\begin{array}{l}\text { Jeudi } \\
\text { 22 septembre 2016 } \\
\text { 13h30-18h00 }\end{array}$ & $\begin{array}{l}\text { Lausanne } \\
\text { World Trade } \\
\text { Center }\end{array}$
\end{tabular}

Atelier consacré à l'informatique au cabinet médical

L'atelier s'adresse aux médecins sur le point d'ouvrir un cabinet médical et aux médecins déjà établis qui veulent changer leur logiciel.

\section{Contenu}

- Attentes à un logiciel pour la gestion du cabinet (Introduction)

- Présentation de six logiciels pour la gestion du cabinet (gestion des données des patients, gestion de la facturation et de l'encaissement, statistiques, gestion de l'agenda, connexion des appareils médicaux au dossier patient, etc.)

\section{Coûts}

Gratuit pour les membres de FMH Services.

\begin{tabular}{lll}
\hline Date & & \\
\hline K28 & Jeudi & Lausanne \\
& 23 juin 2016 & World Trade \\
& 13h30-18h00 & Center
\end{tabular}

\section{Inscription et information}

www.fmhservices.ch ou FMH Consulting Services, Cornelia Fuchs, tél. 0419250077

\section{Remarque}

Les adresses des participants aux séminaires dont les coûts sont couverts en partie ou totalement par des sponsors sont communiquées aux sponsors concernés.

\section{Conditions d'annulation}

Un montant est perçu pour une absence ou une annulation. Il est de:

- 50 CHF par personne dans les 15 jours avant le début du séminaire;

- 100 CHF par personne dans les 7 jours avant le début du séminaire.

\section{Apertura e rilevamento di}

\section{uno studio medico}

Il seminario è destinato ai medici in procinto di aprire o di rilevare uno studio medico.

\section{Contenuto}

- Business Plan (preparazione del piano di finanziamento e del credito d'esercizio, prestito bancario)

- Pianificazione (insediamento, progetto e pianificazione, scelta del mobilio, budget)

- Valutazione di uno studio medico (inventario e goodwill)

- Amministrazione di uno studio medico (interna allo studio, rapporti con la banca)

- (tutte le assicurazioni necessarie interne ed esterne allo studio)

- Passaggio dallo stato di dipendente a quello di indipendente

- Fiscalità

\section{Sponsor}

Diversi sponsor si fanno carico delle spese (si rimanda al sito www.fmhservices.ch).

\begin{tabular}{|c|c|c|}
\hline \multicolumn{3}{|c|}{ Date } \\
\hline K50 & Mercoledì & Chiasso \\
\hline & $\begin{array}{l}13 \text { aprile } 2016 \\
\text { dalle } 14.00 \text { alle } 17.30\end{array}$ & $\begin{array}{l}\text { FMH Consulting } \\
\text { Services }\end{array}$ \\
\hline K51 & Mercoledì & Chiasso \\
\hline & $\begin{array}{l}26 \text { ottobre } 2016 \\
\text { dalle } 14.00 \text { alle } 17.30\end{array}$ & $\begin{array}{l}\text { FMH Consulting } \\
\text { Services }\end{array}$ \\
\hline
\end{tabular}

\section{Studio medico associato}

Il seminario è destinato ai medici in formazione che desiderano esercitare la loro futura attività in uno studio medico associato. Liberi praticanti che auspicano affiliare o che hanno già affiliato il loro studio medico individuale a uno associato.

\section{Contenuto}

- Strategia (obiettivi dello studio medico associato, struttura dell'offerta delle prestazioni)

- Imprenditori (composizione del gruppo di lavoro e regole di condotta, partecipazione finanziaria e metodi di remunerazione)

- Finanze e diritto (assicurazioni, previdenza e patrimonio, forma giuridica, finanze e fiscalità)

- Ubicazione e allestimento dello studio (esigenze dei locali e analisi della situazione concorrenziale, allestimento dello studio e costi)

- Risorse umane (qualifiche, regolamenti e contratti)

- Direzione e organizzazione (struttura e procedimenti, compiti, responsabilità e competenze)

- Informatica e amministrazione (aspettative dal sistema informatico per lo studio, valutazione dei sistemi d'informazione
Sponsor

Diversi sponsor si fanno carico delle spese (si rimanda al sito www.fmhservices.ch)

\begin{tabular}{lll}
\hline Date & & \\
\hline K88 & Mercoledì & Chiasso \\
& $\begin{array}{l}\text { 8 giugno } 2016 \\
\text { dalle } 14.00 \text { alle 17.30 }\end{array}$ & FMH Consulting \\
& Services
\end{tabular}

Iscrizioni e informazioni

www.fmhservices.ch o FMH Consulting Services, Cornelia Fuchs, tel. 0419250077

\section{Osservazioni}

Gli indirizzi dei partecipanti ai seminari, i cui costi sono coperti in parte o completamente da degli sponsor, vengono comunicati agli sponsor interessati.

\section{Condizioni d'annullamento}

Un importo verrà rimborsato in caso di assenza o annullamento. Esso sarà di:

- 50 CHF per persona entro i 15 giorni prima dell'inizio del seminario;

- 100 CHF per persona entro i 7 giorni prima dell'inizio del seminario. 